\title{
"The role of a negative pressure ventilator coupled with oxygen helmet against COVID-19: a review"
}

\author{
Kathiresan Chandrasekaran ${ }^{1}$ (D) $\cdot$ Aadharsha Monikandan Shaji ${ }^{2}$ (i)
}

Received: 15 June 2020 / Accepted: 12 April 2021 / Published online: 22 April 2021

(C) Sociedade Brasileira de Engenharia Biomedica 2021

\begin{abstract}
Background The coronavirus (SARS-COV-2) pandemic has provoked the global healthcare industry by potentially affecting more than 2014 million people across the globe, causing lasting damage to the lungs, notably pneumonia, ARDS (acute respiratory distress 15 syndrome), and sepsis with the rapid spread of infection. To aid the functioning of the lungs and to maintain the blood oxygen 16 saturation $\left(\mathrm{SpO}_{2}\right)$ in coronavirus patients, ventilator assistance is required.

Materials and methods The main purpose of this article is to outline the need 17 for the introduction of a non-invasive negative pressure ventilator (NINPV) as a promising alternative to positive pressure 18 ventilator (PPV) by elucidating the cons of noninvasive ventilators in clinical conditions like ARDS. Another motive is to 19 profoundly diminish the rate of infection spread by the employment of oxygen helmets, instead of endotracheal intubation in 20 invasive positive pressure ventilator (IPPV) or noninvasive positive pressure ventilator (NIPPV) like face masks and high-flow 21 nasal cannula (HFNC).

Result and conclusion The integration of oxygen helmet with NPV would result in a number of notable facets including the 22 degree of comfort delivered to patients who are exposed to various ventilator-induced lung injuries (VILI) in the forms of 23 atelectasis, barotrauma, etc. Likewise, preventing the aerosol-generating procedures (AGP) diminishes the rate of nosocomial 24 infections and providing a better environment to both the patients and the healthcare professionals.
\end{abstract}

Keywords Acute respiratory distress syndrome $\cdot$ Non-invasive method $\cdot$ Negative pressure ventilator $\cdot$ Intermittent abdominal pressure ventilator $\cdot$ Nosocomial infections $\cdot$ Oxygen helmet

\section{Introduction}

The coronavirus (SARS-COV-2) which is responsible for the COVID-19 pandemic leads to life-threatening complications by causing ARDS, followed by viral pneumonia infection, which further results in damage to the alveolar membrane. The key treatment is to maintain the blood saturation oxygen level greater than $92 \%$ for the patients affected with ARDS (Gibson et al. 2020; Silversides and Ferguson 2013; Siegel and Siemieniuk 2020). Studies state that invasive pressure

Kathiresan Chandrasekaran

kathir300799@gmail.com

Aadharsha Monikandan Shaji

aadharsha.manikandan98@gmail.com

1 Department of Biomedical Engineering, Orocura Technologies Pvt Ltd, Coimbatore, Tamil Nadu, India

2 Department of Biomedical Engineering, Sri Shakthi Institute of Engineering and Technology, Coimbatore, Tamil Nadu, India ventilator (IPV) for treating ARDS will both aggravate the lung injury and also lead to multi-system organ failures (Singh et al. 2014; Diamond et al. 2020; WHO 2020a, 2020b; Brochard 2003). Other approaches enumerate that high positive end-expiratory pressure (PEEP) associated with low tidal volume ventilation will result in impaired oxygenation (Pi et al. 2015; Hong et al. 2010; Pesenti and Patroniti 2003). On the contrary, enforcing high PEEP on severe ARDS patients is inadequate to maintain the lung volume (Çoruh and Luks 2014; Pesenti and Patroniti 2003). The motivation of the article is to provide an assistance for adequate oxygenation in the lungs similar to the natural respiratory mechanism without arising any complications in contrast to PPVs (Raymondos et al. 2012). This study elaborates the importance of oxygen helmets in respiratory care wards during the evolution of infectious disease such as COVID-19. After expounding the background on ventilators, the state of the art will also be reviewed to indicate the existing negative pressure ventilation systems' and oxygen helmets' significance in the era of infectious respiratory diseases. Lastly, this article attempts to 
initiate the concept of blending the negative pressure ventilation technique, particularly intermittent abdominal pressure ventilation (IAPV), with oxygen helmet to possibly reduce certain existing problems and has the prospects of becoming an additional resource in the field of respiratory instrumentation. This alternative to positive pressure ventilation technique during the pandemic will serve as a reliable technology to treat the lung conditions like ARDS in a desirable way and also help to control the nosocomial infections during the current COVID-19 pandemic, as well as for the future pandemics.

\section{Mechanical ventilation}

Ventilation in pulmonology is a natural process of the movement of air from the atmosphere to the lungs and vice versa, whereas mechanical ventilation is an artificial process of driving the breathable air into the lungs and moving the expired air into the atmosphere (Pupella 2018; Poor 2018; Tobin 1994). Obstruction to the natural breathing mechanism citing various physiological barriers in a person leads to difficulty in breathing subjecting a person to require the assistance of mechanical ventilation. The initial usage of mechanical ventilators can be traced back to late 1800 s, where the very first mechanical ventilator-Spirophone (1876-1928), a tank ventilator built by Woillez (Hess et al. 2001; Tobin 1994; Slutsky 2015) also known as iron lung (Bahns 2015; Kacmarek 2011) -was in practice to treat complications arising from acute respiratory failure (ARF), chiefly the neuromuscular disorders involved. Since then, the ventilators have played a pivotal role in the field of pulmonology, while having a potential evolution of their own.

\section{Types of mechanical ventilators}

From the time since ventilators were first used, their advancement has had an enormous growth paving way to numerous categories of classification (Fig. 1). However, the common categorisation relies on the basis of the pressure provided which leads to two broad divisions: (i) positive pressure ventilator (PPV) and (ii) negative pressure ventilator (NPV) (Tobin 1994; Corrado et al. 1996; Khandpur 2003). Of these, the PPV is unanimously carried out in hospitals to extend assistance in critical care units (CCU) and in treating respiratory disorders. This has aided PPVs to continually be one among the well-explored and highly sought-after fields of research. With a wide range of PPVs in existence, an outline of classification converging all its types has been attempted. Probing the nature of employment, the PPVs fall under two main types - invasive PPVs (IPPV) and non-invasive PPVs (NIPPV), which further descends to full-support PPVs (CMV) and partial-support PPVs under invasive PPVs and constant airway pressure PPVs and variable airway pressure PPVS under non-invasive PPVs. Delving deeper into the rudiments, each of the above-mentioned classes has divisions of its own as illustrated in (Fig. 1). With regard to the NPVs, their less usage has restricted them with very few advancements and with four major types - the conventional iron lung, cuirass, jacket/bodysuits, and intermittent abdominal pressure ventilation (IAPV) (Lucangelo et al. 2008). The following context explores the less-explored fundamentals and the possible usage of NPVs.

\section{Positive pressure ventilator}

PPV employs surging of a gaseous mixture, specifically with the required proportion of oxygen and a pressure higher than the atmospheric pressure, into the airways of the subject by a tube that delivers it in the intra-alveolar space during inspiration (Soni and Williams 2008). Though originally designed to assist the air force fighters in high-altitude flights during the World War II, the PPV usage accelerated during the polio outbreak in the 1950s mostly in the USA and Scandinavia. Results showed an explicit reduction in the mortality rates of people with respiratory paralysis and polio (Jackson 2019). This marked the beginning of the PPV's remarkable role as a life-supporting device revolutionizing the treatment procedure in CCUs. The paramount importance of PPVs over NPVs has subjected to it various technical advancements and modifications resulting in an array of PPVs. Currently, as the world is exposed to an unpredicted pandemic due to severe acute respiratory syndrome coronavirus 2 (SARSCOV-2), plainly COVID-19 and with no promising vaccine so far, the PPVs act as an ally in treating the virus infected subjects who develop prominent secondary pulmonary infections (Bao et al. 2020; Li et al. 2020a).

The air supply through PPVs is generally carried out by two major mechanisms partially relying on the physiological state and conditional requirements of the treatment planned with primary stages of infections with minimal lung damage being treated with NIPPV and the cases with negligible lung capacity or severe damage being treated with IPPV (Brochard 2003). Inspection of the lungs involving various pulmonary function tests (Ranu et al. 2011; Adams 1997) guides in determining the optimal type of PPV to be provided.

Invasive PPV A typical invasive PPV requires the surgical procedure of tracheostomy, which involves a minor incision at the subject's neck to assist a direct entrance to the patient's airway (Walter et al. 2018; Ahmed and Athar 2015). The incision is commonly made inches above the suprasternal notch transversely ending with the opening of the trachea, the juncture point to facilitate the entry of the endotracheal tube (Engels et al. 2009; De Leyn et al. 2007; K M 2008). Numerous alternative techniques and procedures are available 
Fig. 1 An outline of artificial/ mechanical ventilators
Artificial / Mechanical

Ventilators

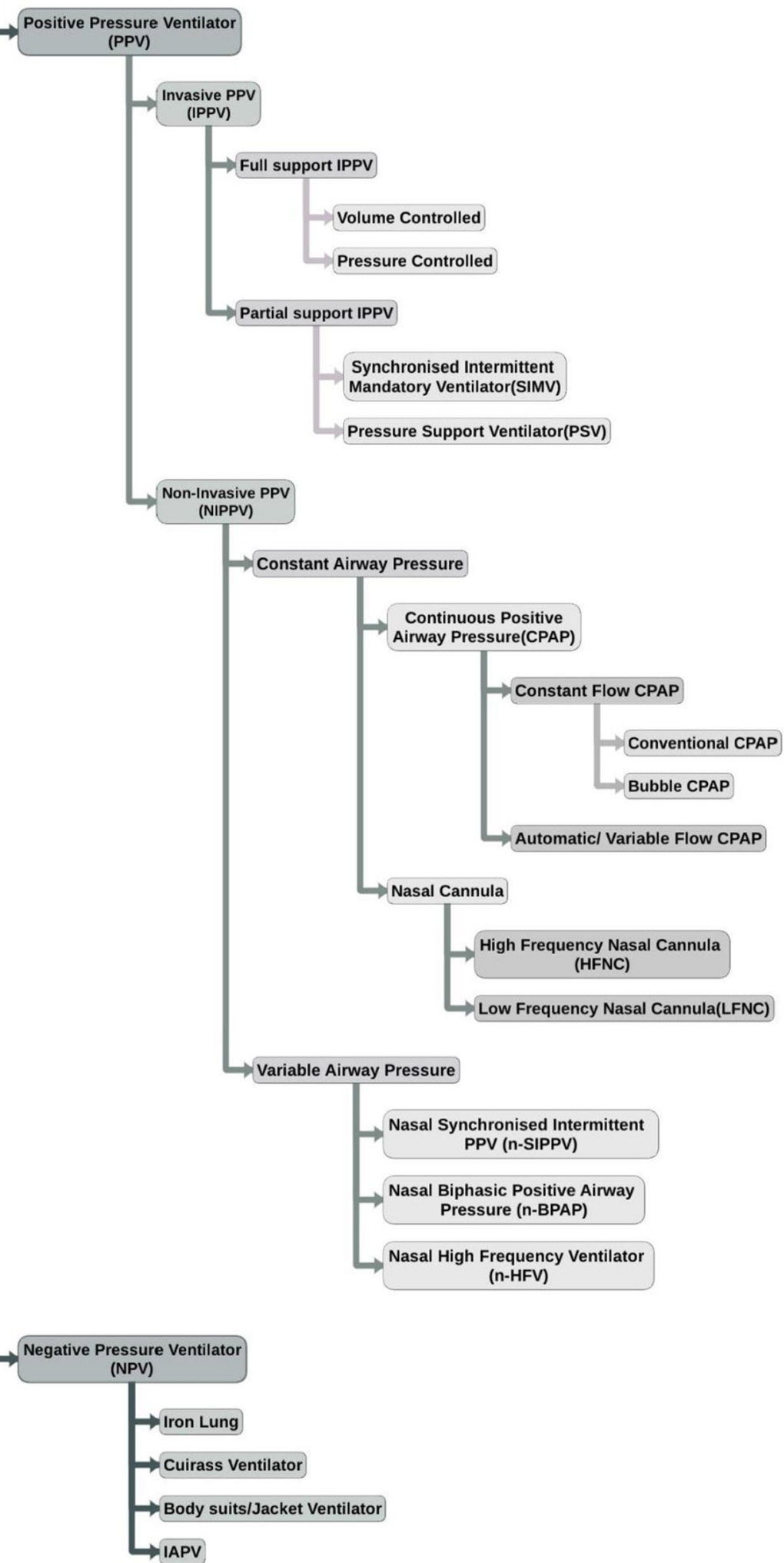


to carry out the same. However, the conventional open surgical tracheostomy (OST) and the more favourable percutaneous dilatational tracheostomy are widely employed (Rashid and Islam 2017). A constant ventilation support for prolonged periods of time is the major reason to conduct tracheostomy and also other reasons like emergency access, blockages, and failure in trachea-bronchial fluid clearance. As the spread of COVID-19 began globally in the beginning of 2020, the hospitals across the world witnessed a substantial influx of patients requiring extensive ventilation support. Normally, 8$13 \%$ of ICU ward patients require IPPV support (McGrath et al. 2020). The additional attributes of COVID-19 such as laryngeal oedema, airway obstruction, and extubation failure force the existing standards of practice to settle for tracheostomy, thus raising the rate of implementation. On the basis of severity, the ventilator support has been modified into a couple of subdivisions.

i. Full-support PPVs are used when certain lung conditions require a complete and continuous takeover by the mechanical ventilation for support and recovery which presents continuous mode or full-support ventilators. In tertiary stages of lung infections or during lung failures, the physiological functioning of the lungs is entirely replaced by this type of ventilators. It operates regardless of the natural mechanism by delivering a preset volume of air in regular intervals. The full-support CMV enables pressure-controlled or volume-controlled modes. Volume-controlled ventilation is the initial ventilation mode particularly in CCUs and emergency departments (ED) as it delivers a constant volume of air with a varied pressure that helps monitor and maintain pulmonary compliance and airway resistance. However, barotrauma poses an intruding risk (Kumar et al. 1973; Diaz and Heller 2020; Ioannidis et al. 2015). The pressure controlled, though reduces the barotrauma (Kumar et al. 1973; Ioannidis et al. 2015; Chacko et al. 2015), finds limited usage in CCUs and EDs as it demands close monitoring. It works by varying the tidal volume and compliance by a preset pressure value that begins once peak inspiratory pressure (PIP) is attained. Precisely, volume-controlled ventilation and pressure-controlled ventilation are just the different control variables which are present in a conventional ventilator and based on patient needs, the control variable will be selected for respiratory support.

ii. Partial-support IPPVs were developed to assist the partially functioning lungs to perform up to its complete capacity. They try to activate the natural mechanism of inspiration by corresponding with the patient's spontaneous efforts, particularly in acute phases of respiratory disorders. The synchronous intermittent mandatory ventilation (SIMV) has preset mandatory breaths delivered in synchrony with the patient's effort determined during a window of time. Greater improvements have been incorporated in terms of dynamic hyperinflation, patient comfort, and barotrauma. However, the major flaw of this design being the increased work of breathing (WOB/ breath) (Lazoff and Kim 2019; Downs et al. 1973). Another partial-support ventilation for spontaneously breathing patients, which lets the patient be in charge of the respiration rate, volume, and the flow rate is the pressure support ventilation (PSV). With a preset pressure value, this maintains airway pressure until the cut-off is reached. The PSV in combination with SIMV has shown great results in considerably decreasing WOB to normal range (Jounieaux et al. 1994).

With the outbreak of novel coronavirus 2 (SARS-COV-2), studies reveal that acute respiratory distress syndrome (ARDS) has sustained a stable prevalence among the patients. The partial-support ventilators offer a low-level treatment in the preliminary stages of ARDS (McMullen et al. 2012). Compared to other PPVs, it shows promising results in terms of haemodynamic function and oxygenation. However, the procedure requires sedation and affects the pattern of breathing and upsets the state of dead space (Henzler et al. 2006).

Invasive PPVs though being an incredible instrumentation in battling have their share of shortcomings in terms of tracheal tube infections, respiratory tract infections due to upper airway reflexes, sedation, surgical pain, inflammation, inability to speak, and patient discomfort (ESICM Academy 2020). These pile up along with the existing illness to necessarily cause more pain for recovery of the patient.

Non-invasive PPV Administering an alternate amount of pressure depending on the breath cycle via a face mask or nasal cannula is the NIPPV mechanism. It effectively replaces the invasive tube and the surgical procedure of invasive PPVs providing equivalent results thus gaining momentum in wide usage from critical care units (CCU) to homes evolving in different forms according to their place and phase of usage [Borel et al. 2019; Díaz Lobato and Mayoralas Alises 2013; Scala and Pisani 2018). It has been highly utilized in acute phases of respiratory disorders, primarily to treat chronic obstructive pulmonary disorder (COPD) (Duiverman 2018), hypoxaemia (Moerer and Harnisch 2016), acute respiratory failure (ARF) (Brochard et al. 2002), sleep apnoea (Nicolini et al. 2014), pulmonary oedema (Bello et al. 2018), and also reducing the risk of ventilator-associated pneumonia (VAP) (Hess 2005) associated with the invasive ventilation. With respect to the COVID-19 pandemic, the US Food and Drug Administration presented a suggested list of supportive device to fight against the coronavirus infection, wherein the NIVs were asked to be used with incorporation of proper filtration for 
reducing the chance of disease transmission (USFDA 2020) which also increases the use of NIVs.

On the basis of the pressure delivery, NIV are classified into two main classes: (i) constant airway pressure NIVs and (ii) variable airway pressure NIVs.

i. Constant airway pressure (CAP) ventilator functions by applying a continuous invariable pressure to aid the airway from being unobstructed, combined with spontaneous efforts of the patient. The frontiers of medical industries have designed numerous CAP devices that are simple, economic, and efficient in delivering quality healthcare (Pinto and Sharma 2020). Deployed highly in neonatal CCUs (DiBlasi 2011) and for sleep apnoea, the CAP usage has shown significant reduction in death rates associated to the same (Batool-Anwar et al. 2016; Spicuzza et al. 2015; Lettieri et al. 2020). The initial and commonly used type of CAP is continuous positive airway pressure (CPAP). The components include a flow initiator, a face mask and a tubular hose sometimes with a humidifier (Pinto and Sharma 2020). With a single principle of prevention of obstruction, the CPAP functions as the most simple yet an efficient support system that provides placid pressure to subjects by far, being the most resourceful therapy for sleep apnoea(Batool-Anwar et al. 2016; Spicuzza et al. 2015). Since the pressure is constant, variation in flow is sometimes done based on the treatment parameters. This leads to an extra classification of CPAPs into constant flow and variable flow CPAPs with constant flow CPAPs further leading to a subtype-bubble CPAPs (Yagui et al. 2011). These CPAPs are designed with particular interest in neonatal care, where implementation of masks would be risky. The variable flow CPAPs referred to as automatic positive airway pressure (APAP) have algorithms that help in varying the flow accordingly (Yagui et al. 2011).

Although CPAPs have their set of advantages, a slight set of troubles are posed by them such as mechanical difficulties, nasal septum trauma, long-term wearing, intolerance with patient movement etc. These are rectified by a more sophisticated yet similar equipment- the nasal cannula. Unlike CPAP, it comes with a nasal tube with prongs to aid the flow of oxygen. Categorized into high-flow nasal cannula (HFNC) and low-flow nasal cannula (LFNC), it is often used for oxygen flow therapies based on their flow. Notably, HFNCs are effective in improving oxygenation in COVID-19 patients (Wang et al. 2020). However, HFNCs are highly represented as aerosol-generating procedures due to its high gas flow (Li et al. 2020b).

ii. Variable airway pressure ventilator (VAP) is similar to CAP in context. However, VAP exhibits one change in terms of the pressure of the gas expelled. Unlike the CAPs with a pre-set pressure, the VAPs alter their pressure according to the patient's spontaneous efforts. Being the prime members of the PPV evolution chain, they are refined with a set of formulae to calculate the pressure requirements (Spieth et al. 2009). The commonly used VAPs include Nasal Intermittent PPV (NIPPV) which is employed in infants surpassing nasal continuous positive airway pressure (n-CPAP). It operates by superimposing an intermittent peak pressure on it. It has improved the nasal septal erosion and obstruction prevailing in $n$ CPAP. Nasal bi-level positive airway pressure (nBPAP) employs a combination of inhalation- and exhalation-prescribed pressure. This dual pressure combination helps the patient to get better breathing experience. Like NIPPV, it is used for CPAP failed patients with low oxygen level, sleep apnoea, congestive heart failure, and neuromuscular disorder (Lemyre et al. 2016). Another interesting modern method of ventilation is the usage of high-frequency ventilation (HFV). The HFV provides a considerably exceeding number of breaths per minute in comparison to normal breath per minute (bpm). For example, $900 \mathrm{bpm}$ is provided for infants. It has 3 principal types-high-frequency positive pressure ventilation (HFPPV), high-frequency jet ventilation (HFJV), and HFOV. They are shown to have reduced barotrauma, better blood gas management with minimal tidal volume (TV) utilization, and with decreased morbidity in respiratory distress syndrome management (Ellsbury et al. 2002).

Though the non-invasive ventilators have made massive changes to the shortcomings of invasive PPVs, they still have a few of their own and these include the uncomfortable interfaces, possibilities of air leak, time-consuming, acidosis, severe hypoxia, not compatible with physical restraints, aerophagia

, aspiration and nausea, unsuitable for gastro-intestinal tract surgical patients, and secondary infections due to improper clearance of secretions (Raymondos et al. 2012; Yarstev 2015; Carron et al. 2013).

\section{Negative pressure ventilator}

Periodic generation and application of negative pressure to a sealed portion of the body to stimulate the breathing process is negative pressure ventilation in a nutshell. The expansion of lungs and chest is initially achieved by the negative pressure exertion upon the subject's body in order to aid a patient achieve natural respiratory mechanism. This causes the circulation of air to enter the lung cavity and the removal of the negative pressure aids in natural contraction with consequent exhalation. Certain cases are provided with assistance for inhalation and exhalation (Shneerson 1991; Grum and Morganroth 1988; Jackson and Muthiah 2019). Even though they had an origin similar to the PPVs, their usage was much 
restricted, with limited engagement in neuromuscular and skeletal disorder treatments with PPVs replacing their role. They have a habit of becoming a topic of research and practice during pandemic periods, for their last known extensive usage was during the polio pandemic of 1950 (Shneerson 1991) and are currently being used and researched prevalently to fight the novel coronavirus (SARS-COV-2). Being a less pondered subject, the progression of NPVs is comparatively less than the PPVs.

The chiefly notable NPVS are the following four:

- Iron lung

- Cuirass ventilator

- Jacket/bodysuit ventilator

- IAPV

Apart from these, the COVID-19's immense need of ventilators to support the patients and the shortage of positive ventilators have led to an increased research in the role of negative pressure ventilation with a handful of new and improved negative pressure technologies coming up (CameronChileshe 2020).

Iron lung The foremost NPV-iron lung, also called as tank ventilator, was set up at Harvard University in 1928. It has a chamber/tank commonly made of aluminium or plastic which seals the entire body except the head. Within the chamber, the pressure changes are made enabling vacuum inside that triggers the breathing (Fig. 2). A mattress with neck rest, being a part of the design, ensures ideal patient positioning. Facilities to observe and operate patients are available which help in blood gas monitoring and physiotherapy. They also possess rotary pumps to eliminate the air within the chamber. With many models emerging on the same principle, the most commonly used model was 'the alligator' (Shneerson 1991; Jackson and Muthiah 2019; Antonaglia et al. 2008). Their size, inability to port, cost, and aspiration tendency clouded

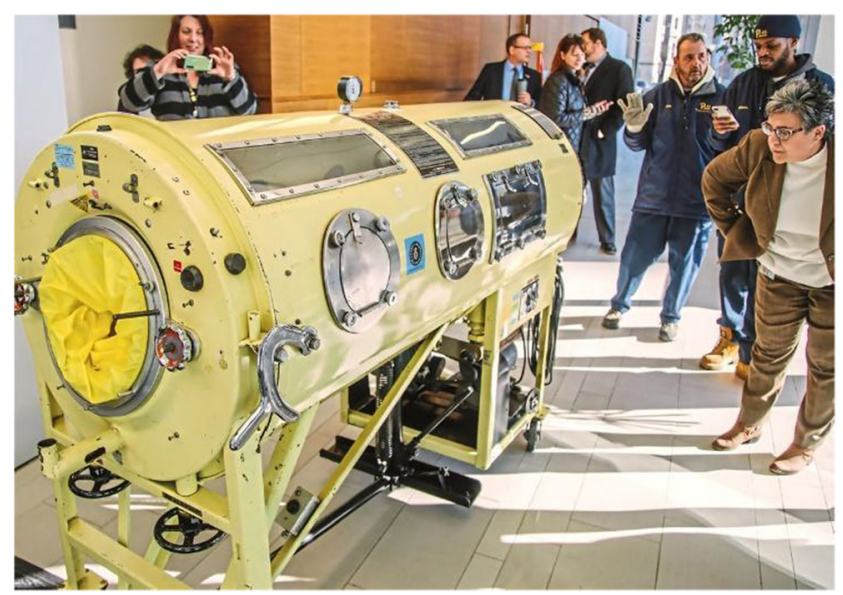

Fig. 2 Iron Lung. (Used with permission from "University of Pittsburgh) its efficiency and very minimal requirements to assist the patient breathing. However, lighter model iron lungs have been designed since then. An improvisation in size and cost of these is being attempted in various parts of the globe to substitute the PPVs amid the COVID-19 pandemic.

Cuirass ventilator The cuirass ventilation is facilitated with a closed casing that encloses the chest and abdominal region within it and has a back strap patient attachment. It is accompanied by a hose inlet that paves way for the negative pressure mechanism to work. Earlier designs of cuirass ventilators were incompetent due to their modelling flaws mostly being misfit. Nevertheless, with close attention to details involved in manufacturing, the flaws were rectified, with customized casts with proper fitting and airtight seals with edge padding and an extra airtight covering. From the cast out of plaster of Paris, the casing is moulded. Synthetic materials like vitrathane or fibreglass are used for they weigh light and are airtight. Their shortcomings were in terms of misfits, air leak, and development of pressure contact points on the enclosed body surface. With the technological growth, these have been overcome, thus presenting a robust, durable, cost-effective, light, and easy to use as an alternative for ventilators. It is very similar to nasal intermittent PPV and is also available in controlled form and assistive form. They have been used in infants to treat bronchiolitis obliterans (Shneerson 1991; Antonaglia et al. 2008).

Cuirass ventilation coupled with a combination that controls both the inhalation and exhalation phases is collectively referred to as biphasic cuirass ventilation. This method has gained massive momentum in this COVID-19 pandemic for they act as suitable alternatives in many ways.

Jacket ventilator The development of Jacket ventilators was an improvement planted to replicate the functioning of Cuirass ventilators without the shortcomings, particularly the misfit concern. The initially developed jacket ventilator was the Tunnicliffe jacket. The fundamental layout of the jacket ventilator has an airtight synthetic garment lined with an inner framework of plastic or metal grid, a suction pump and a back plate until the hips. They are highly employed in home usage compared to hospital usage for their efficiency is less compared to other treatment methods. However, they are preferred less by the patients due to interfacial discomforts, cost, cumbersome nature, and intolerance to withstand pressure. But it does not rule them out of being a substitute to PPVs in critical times. Certified jacket ventilators such as Lifecare Pulmowrap, Poncho Wrap, Nu Mo suit, Pneumo Wrap, Pneumo suits, and Zip Suits are extensively suggested for home care rather than long-term hospital usage (Shneerson 1991; Antonaglia et al. 2008).

Intermittent abdominal pressure ventilator It is a method that employs positive expiratory pressure outside the patient's 
body to promote the natural breathing mechanism. An inflatable bladder-laden belt is placed around the subject's abdomen, which upon inflation forces the internal contents including the diaphragm to undergo an upward displacement which consequently advances exhalation. Upon the pressure removal in the belt, the internal environment returns to its normal state and enables inhalation (Fig. 3). The ease of use of the belt in matters of portability, positioning, and long duration usage put this in the row of possible NPV substitutes despite of having drawbacks concerning patient angle requirements $\left(>30^{\circ}\right.$, optimally at $75^{\circ}$ ), breathing synchronization and inefficiency (Antonaglia et al. 2008; Banfi et al. 2019).

In spite of having a few drawbacks, particularly in lung compliance, the negative pressure ventilators have some magnificent life-saving features, which are often less noticed. Their statistical records on treating ARF, long-term COPD, paediatric pneumonia, bronchopulmonary disorders, neuromuscular disorders, and cardiorespiratory complications definitely suggest they have a more fruitful scope for research and development (Shneerson 1991; Antonaglia et al. 2008). COVID-19 poses numerous complications to the patients, which are not intended to be further stimulated with additional consequences, and thus, it is believed that the research on NPVs would be of appropriate sense in this stage.

\section{Significance of NPV over PPV against ARDS}

Positive pressure ventilation has been the primary recommendation for the people suffering from ARDS. In mechanical ventilation, the recommended positive end expiratory pressure (PEEP) could minimize the tidal lung stress and strain with better arterial

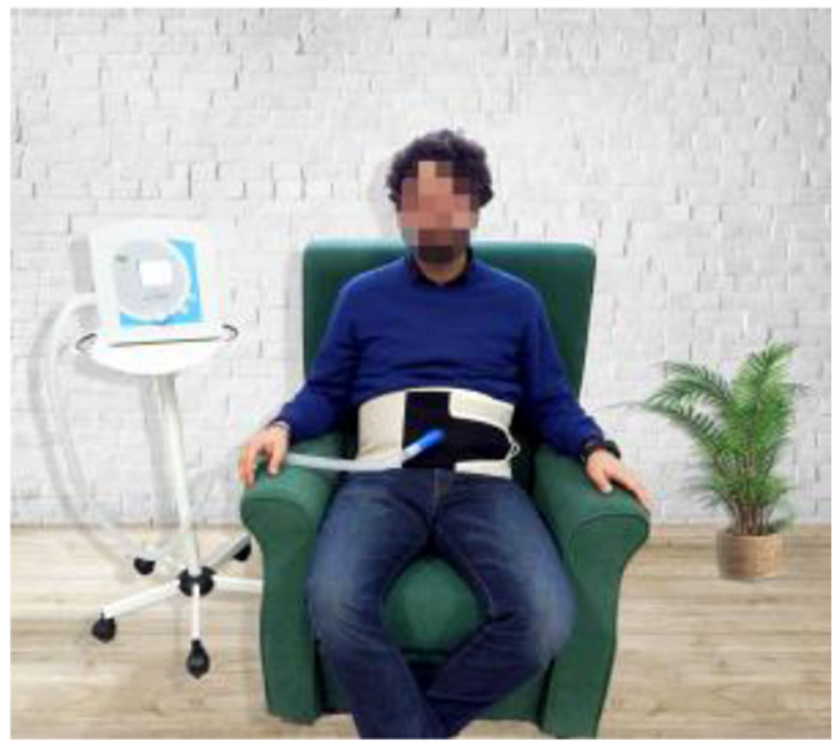

Fig. 3 An example of intermittent abdominal pressure ventilation. (Used with permission from Dr. Francesco Didonna, Dima Italia Srl) oxygenation. However, it contributes to ventilator-induced lung injury (VILI) (Oeckler and Hubmayr 2007) by inducing barotrauma in the lung parenchyma. Various studies also enumerate that high pressure to lung parenchyma could result in pneumothorax. Also, other studies explained that these kinds of positive pressures will result in inflammation which further leads to biotrauma - a multiorgan failure, pneumomediastinum, pneumoperitoneum, and subcutaneous emphysema (Sahetya et al. 2017).

On the other side, low positive pressure could also worsen the lung injury associated with ARDS by impairing the pulmonary surfactant function, which results in atelectasis. A recent study states that the COVID-19 patient's pneumonia pattern is quite different from a typical ARDS pattern. So as to apply a modified mechanical ventilation, with respect to tackle the revamped ARDS pattern, rather than following the traditional mechanical ventilation protocol in ARDS (Tsolaki et al. 2020). Upon weighing the drawbacks of both PPVs and NPVs, the NPVs are found to exhibit fewer limitations as discussed (Raymondos et al. 2012).

\section{COVID-19 and nosocomial infections}

The novel coronavirus (SARS-COV-2) responsible for affecting more than 8 million people around the globe spreads from a person to person by the transmission of respiratory droplets. These infectious droplets reach the mucosal lining/ conjunctiva of a normal person by actions such as coughing, sneezing, talking or touching and it leads to a number of severe respiratory disorders. The transmission also occurs through fomites in the immediate environment around the infected person (WHO 2020a, 2020b). Although COVID-19 infection is not an airborne one, under some exceptional circumstances, there is a possibility for airborne transmission of the virus, specifically during aerosol-generating respiratory support treatments like endotracheal intubation, open suctioning, tracheostomy, non-invasive positive pressure ventilation, and manual ventilation before intubation.. A recent study about the survival rate of the SARS-COV on aerosols revealed that SARS-COV-2 is higher compared to the SARSCOV-1 (Van Doremalen et al. 2020). The aerosol-generating procedures hike the transmission of infection by aerosolizing the virus, which in turn enters the system of healthcare practitioners or a person who comes in contact with the aerosol subsequently increasing the demand for highly safe personal protective equipment (PPE). Also such procedures lead 3 stages of complications ranging from bleeding, subcutaneous emphysema, oesophageal damage, skin rashes, sores, embolism, erosion, development of bumps which increases the chances of infection to a higher scale, leading to further transmission risks. Consequently, this leads to an amplification in need of treatment units and ICU beds. Another study on nosocomial infection demonstrates that the mortality of COVID- 
19 patients with nosocomial infection was $15.4 \%$ higher than the patients without it (He et al. 2020).

\section{Role of oxygen helmet}

The COVID-19 patients with clinical syndromes such as pneumonia, ARDS, and sepsis often require artificial oxygenation support due to reduction in gas exchange mechanism in lungs. While most of the countries are racing to manufacture enough ventilators to meet the demand, most of the respiratory care experts believe that non-invasive oxygen therapy could be made to meet the requirement of a mechanical ventilator. A mechanical ventilator has the ability to take over the complete functioning of lungs through intubation with the requirement of sedatives and delirium, whereas oxygen therapy simply administers the oxygen through a face mask or nasal cannula. As per NIH guidelines, it is recommended to use non-invasive methods to avoid intubation under possible conditions (NIH 2020). Mechanical ventilators are being used on COVID-19 patients when the requirement of oxygen is above 501 per min, and those who require oxygen below this range would generally be treated with HFNC (Nishimura 2015). Although this kind of non-invasive HFNC oxygen therapy greatly eliminates the problems associated with intubation, findings state that helmet-based NIV has shown improved oxygenation than HFNC (Grieco et al. 2020). The helmet-based ventilation initially studied in 2016 and later on researched have shown significant changes to patients with ARDS. Upon delving further into the statistics, oxygen helmet produces results better than the conventional face masks and nasal prongs. A study shows that helmet-based ventilation has a significant reduction of intubation rates than other non-invasive methods and also significantly reduces the complications arising in the ARDS survivors (Patel et al. 2016; Patel et al. 2018). These kinds of oxygen helmets provide the pressurized oxygenated air into the helmet to keep the airway tract open for improving the oxygenation (Easton and Wood 2020). As the oxygen helmet entirely seals the head of an affected person, it highly eliminates the potential causes of aerosolization which is the common disadvantage of respiratory support devices. The helmet-based ventilation (Fig. 4) was tested against face masks, and it was concluded that the intubation period was significantly low for people with helmets and also the air leaks were negligible. The research showed $61.5 \%$ intubation for face mask whereas the helmet required a period of $18.2 \%$. The deaths and adverse effects were comparatively less compared to the face mask ventilation (DiBlasi 2011).

\section{Combined effect of NPV and oxygen helmet}

NPVs were the principle respiratory support devices during the epidemic of poliomyelitis in the 1950s (West 2005). Later, due to

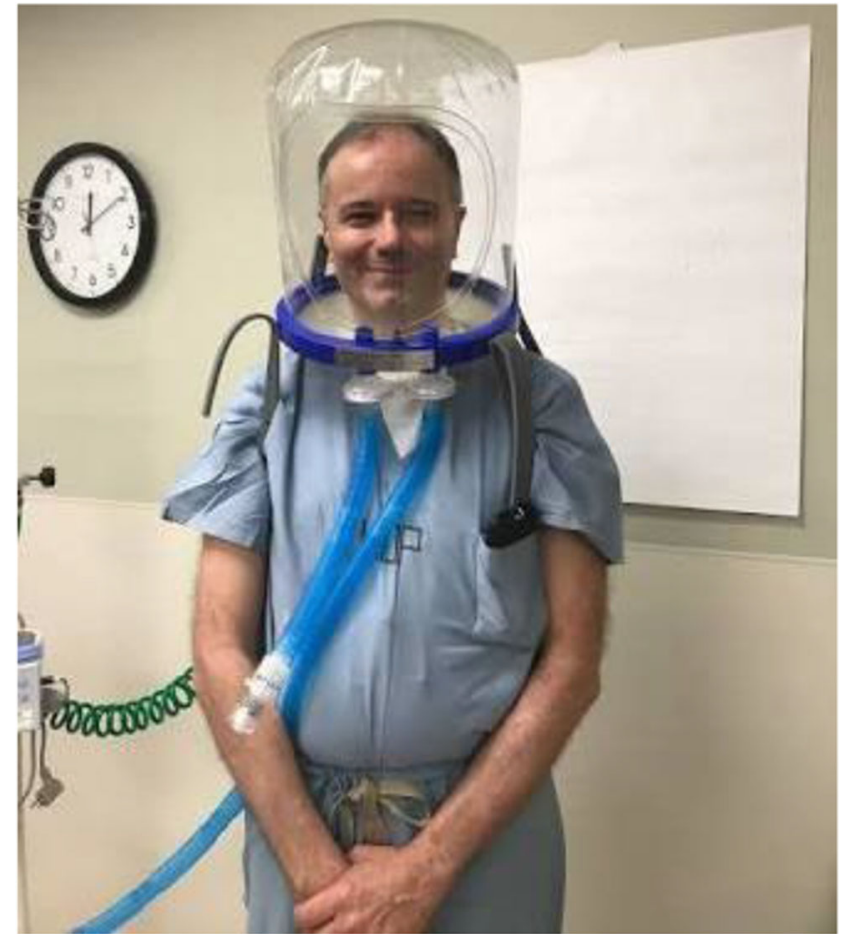

Fig. 4 Helmet-based ventilation. (Used with permission from the author, Dr. Maurizio Franco Cereda MD, Department of Anaesthesiology and Critical care, University of Philadelphia)

the rapid innovations and advancements in PPVs, NPV's exploration was limited, though it has numerous advantages over PPVs, its disadvantages such as bulky, hypoxemia during sleep due to upper airway obstruction (Cheifetz and Wetzel 2015), and motion restriction were highly noticeable. On comparing various studies, it is clear that NPVs have fewer limitations when compared to PPVs. Various researches on NPVs are recently being carried out to overcome its fewer drawbacks, which leads to the innovation of biphasic cuirass ventilator, Exovent (Fig. 5) and IAPVs by overcoming the cons in a typical iron lung ventilator. As discussed previously, IAPVs have numerous advantages in treating ARDS, except the disadvantage of low lung compliance, though the compliance is better than iron lung ventilator. This low lung compliance can be overcome by using the oxygen helmet and IAPVs simultaneously. This combinational effect could be a potential therapy in treating the clinical syndrome like pneumonia, ARDS, and sepsis in COVID-19 pandemic. Introduction of oxygen helmets are not only to support the IAPV system but also to provide multiple advantages in controlling the disease transmission to the healthcare professionals, thus reducing the rate of nosocomial infection.

\section{Discussion}

The extensive study conducted here to analyse the role of negative pressure ventilators in fighting the COVID-19 pandemic 


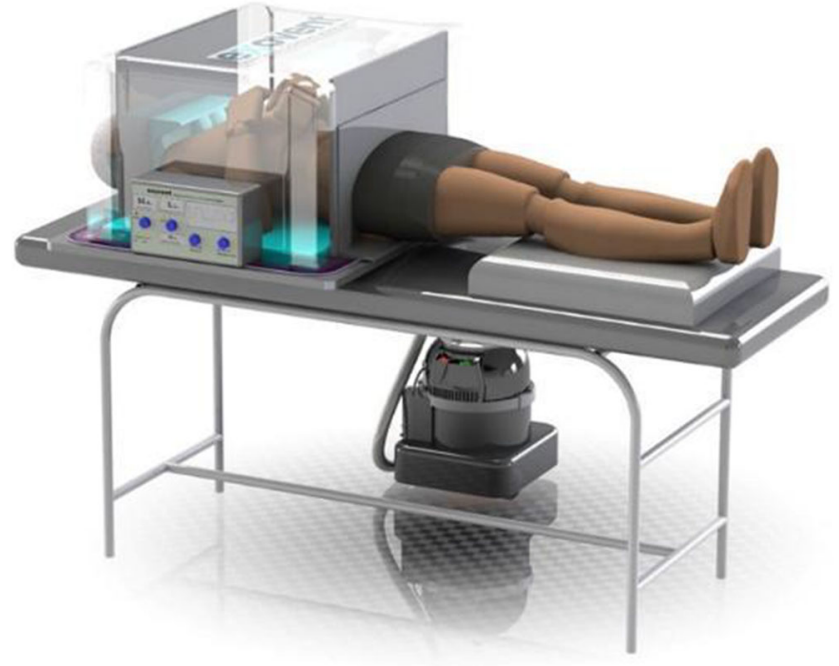

Fig. 5 Exovent - a recently developed negative pressure ventilator. This is the state of the art which is currently being presumed to treat COVID19 due to its potential advantages over positive pressure ventilators. (Used with permission from "Exovent" company)

helped us journey through the various existing and primordial ventilators that existed before the significant ventilator evolution in the mid-late twentieth century. This expedition has flourished us with valuable insights that are guided in learning the working of ventilators which differs with each case requiring a specific set of functionalities. The ventilators, though playing a vital role as a supporting device in CCUs, do have an interesting facet in regard to therapeutic effects. Emerging as the heroes of the world's battle against the coronavirus (SARS-COV-2), they have definitely helped their share in providing critical care and support to victims and increasing the recovery rate. However, their limitation in number with the rapidly growing demand has made an alarming call for alternatives to support them (Kliff et al. 2020). At this point, manufacturing a substantial number of them would consume time and money, thus affecting the global economy. This has been identified as a worldwide crisis and researchers across the globe are on lookout for remedial measures. As the studies reveal that PPVs are the predominantly used ventilators, they do have a number of limitations which adds a strain on their common usage (ESICM Academy 2020; Yarstev 2015; Carron et al. 2013). To put an end to this upsurging need with a suitable alternative, we have come up with an alternative based on the NPVs and helmet ventilation by evaluating their functionalities, with the help of previous research around the world. A combinational application of intermittent abdominal pressure ventilation (IAPV) with the oxygen helmet would be a promising alternative for PPVs against COVID-19 and ARDS, a key companion of COVID-19. These medical modalities complement each other's insufficiency while considerably delivering a successful ventilator support in patients in need of support. As the IAPVs are simply belts with inflatable bladders within and are worn as a belt, this design considerably reduces a lot of limitations posed by the conventional ventilators (Fig. 3). It is ideal in parameters concerning long-term usage, lightweight, stealth operation, portability, cosmesis, etc. thus qualifying as a respiratory support device (Banfi et al. 2019). However, the odds of fighting against COVID-19 or any other acute respiratory disorder yearn more, where the IAPV alone would be insufficient due to its efficiency lag (Antonaglia et al. 2008). With very few advancements over the years, IAPVs still face an efficiency lag. The focus on overcoming the IAPVs cons has been directed to the exploration of oxygen helmets, a support system that enhances the efficiency of IAPVs by synchronizing the patient's breathing pattern properly. Oxygen helmet could be used not only for aiding IAPVs but also for reducing the rate of threatening nosocomial infection, as it entirely covers the respiratory openings of an ill person. Additionally, we had a major check on ease of use, as well as the production of the suitors and predicted the helmet-based ventilation, qualified our constraint on cost, ease of use, and production. With the global ventilator desperation, this combinational ventilatory assistance was derived with conclusive evidence based on various researches. In previous studies (Raymondos et al. 2012) between PPV and NPV in treating ARDS, the usage of NPV has shown an improved gas exchange than PPV with slightly improved initial haemodynamics. In one hour, the $\mathrm{PaO}_{2}$ : $\mathrm{FaO}_{2}$ ratio (mean arterial to inspired $\mathrm{O}_{2}$ ratio) showed an increment by $40 \%$ ( $92 \mathrm{mmHg}$ ), and by the second hour, it was $30 \%$ (76 $\mathrm{mmHg}$ ), thus decreasing pulmonary shunting as well. Even though the lung volumes remained the same, the intra-abdominal pressure decreased in NPVs (Raymondos et al. 2012; Easton and Wood 2020). The study conducted by a group of Russian physicians concluded that NPVs can be used with clinical effectiveness. Various research evidences as discussed above prompted us to opt for a combinational therapy, of helmet-based ventilation and IAPV, which might aid in facing the towering ventilator demand. Due to lack of laboratory resources and lockdown activities in amendment due to COVID-19, further research down this lane have not been carried out. The limitations in this model may chiefly arise with the tubular inflation cycle, neck sores, claustrophobic intolerance, air leak management and other minor drawbacks. However, since the NPV field is currently being a dynamic field with innovative ideas surfacing every day like the Exovent (Fig. 5), modern iron lungs, and biphasic cuirass ventilators, these limitations can be overcome and a novel ventilator model's possibility is also present (Cameron-Chileshe 2020). In regard to the situation prevailing currently, with the number of cases soaring each day, this combinational therapy of IAPV and helmet-based ventilation has the potentiality to improve the state of lives at stake.

\section{Conclusion}

As the entire world is plunged into the research of all kinds to fight against the coronavirus (SARS-COV-2), this article 
contributes to the same by examining an intriguing perspective of NPVs and helmet-based ventilation. These two respiratory support procedures are combined to achieve a credible level of lung compliance, as it is a major concern with respect to the limited use of NPVs. In order for NPVs to attain this required level of lung compliance, the oxygen helmet provides extensive support with its highly beneficial attributes. The ideology here attempts to widen the ongoing discussion and experimentation on NPVs' comeback. Though adequate assessments are yet to be conducted, a unified operational test is believed to increase its importance among the class of treatments available. Accuracy and synchronization, being the two key parameters of the proposed system, are expected to be improved with further research and its assessments. Rising technologies mediate the deficiencies of NPVs and the minor limitations can be resolved with appropriate auxiliary or combinational devices. With the mainstream research being conducted with the PPVs, further research should be carried out in NPVs to exploit their role in the healthcare industry. Studies have begun propagation since the onset of COVID-19 pandemic, yet it remains an understudied sector with a wide scope. These considerations, upon right assessment, shall lead to abundant resources that will help in preparing ourselves for such pandemics and contribute to the recognition of different control measure implementation.

Acknowledgements Thanking Dr. T. Gayathri, Ph.D, and Dr. G.C. Sowparnika, Ph.D_professors in the Department of Biomedical Engineering - for their guidance throughout the article as a consultant and making several critical revisions of the work.

Code availability Not applicable.

Author's contribution 1. Kathiresan Chandrasekaran, corresponding author, has made substantial contributions to conception, data analysis and interpretation, drafting the work, and critical revision of it.

2. Aadharsha Monikandan Shaji has made contributions in data interpretation and in drafting.

Data availability Not applicable.

\section{Declarations}

Conflict of interest The authors declare that they have no conflicts of interest.

Ethical approval Not applicable.

Consent to participate and consent for publication Not applicable.

\section{References}

Adams A. Pulmonary function in the mechanically ventilated patient. Respiratory Care Clinics of North America. 1997;3(2):309-31.
Ahmed S, Athar M. Mechanical ventilation in patients with chronic obstructive pulmonary disease and bronchial asthma. Indian Journal of Anaesthesia. 2015;59:589. https://doi.org/10.4103/0019-5049. 165856.

Antonaglia V, Pascotto S, Piller F. Advanced modalities in negativepressure ventilation. In: Pelosi P, Zin W, Aliverti A, editors. Lucangelo U. Respiratory System and Artificial Ventilation: Springer; 2008. p. 221-35.

Bahns E (2015) It began with the Pulmotor the history of mechanical ventilation. Draeger.

Banfi P, Volpato E, Bach J. Efficacy of new intermittent abdominal pressure ventilator for post-ischemic cervical myelopathy ventilatory insufficiency. Multidisciplinary Respiratory Medicine. 2019;14. https://doi.org/10.1186/s40248-019-0169-4.

Bao Z, Gu B, Liu J, Zhu J, Alffenaar JW, Hu Y, et al. Successful treatment of patients severely ill with COVID-19. The International Journal of Tuberculosis and Lung Disease. 2020;24:650-3. https:// doi.org/10.5588/ijtld.20.0232.

Batool-Anwar S, Goodwin J, Kushida C, et al. Impact of continuous positive airway pressure (CPAP) on quality of life in patients with obstructive sleep apnea (OSA). J Sleep Res. 2016;25:731-8. https:// doi.org/10.1111/jsr.12430.

Bello G, De Santis P, Antonelli M. Non-invasive ventilation in cardiogenic pulmonary edema. Annals of translational medicine. 2018;6: 355-5. https://doi.org/10.21037/atm.2018.04.39.

Borel J, Palot A, Patout M. Technological advances in home non-invasive ventilation monitoring: reliability of data and effect on patient outcomes. Respirology. 2019;24:1143-51. https://doi.org/10.1111/ resp.13497.

Brochard L. Mechanical ventilation: invasive versus noninvasive. Eur Respir J. 2003;22:31s-7s. https://doi.org/10.1183/09031936.03. 00050403.

Brochard L, Mancebo J, Elliott M. Noninvasive ventilation for acute respiratory failure. Eur Respir J. 2002;19:712-21. https://doi.org/ 10.1183/09031936.02.00295502.

Cameron-Chileshe J. Iron lung' backed by Stephen Hawking's family to be trialled on Covid-19 patients. In: The Daily Telegraph. 2020 https://www.telegraph.co.uk/news/2020/04/11/iron-lung-backedstephen-hawkings-family-trialled-covid-19-patients/?WT.mc_id= e_DM1233282\&WT.tsrc=email\&etype $=$ Edi_GHS_New Daily\&utmsource $=$ email\&utm_medium $=$ Edi_GHS_New Daily20200411\&utm campaign=DM1233282. Accessed $2 \overline{5}$ May 2020.

Carron M, Freo U, BaHammam A, et al. Complications of non-invasive ventilation techniques: a comprehensive qualitative review of randomized trials. Br J Anaesth. 2013;110:896-914. https://doi.org/10. 1093/bja/aet070.

Chacko B, Peter J, Tharyan P, et al. Pressure-controlled versus volumecontrolled ventilation for acute respiratory failure due to acute lung injury (ALI) or acute respiratory distress syndrome (ARDS). Cochrane Database Syst Rev. 2015. https://doi.org/10.1002/ 14651858.cd008807.pub2.

Cheifetz I, Wetzel R. Negative pressure ventilation. In: parent R (ed) pulmonary hemodynamics- comparative biology of the Normal lung, 2nd ed. science direct; 2015. pp 205-243.

Corrado A, Gorini M, Villella G, De Paola E. Negative pressure ventilation in the treatment of acute respiratory failure: an old noninvasive technique reconsidered. Eur Respir J. 1996;9:1531-44. https://doi. org/10.1183/09031936.96.09071531.

Çoruh B, Luks A. Positive end-expiratory pressure. When More May Not Be Better Annals of the American Thoracic Society. 2014;11:132731. https://doi.org/10.1513/annalsats.201404-151cc.

De Leyn P, Bedert L, Delcroix M, et al. Tracheotomy: clinical review and guidelines. Eur J Cardiothorac Surg. 2007;32:412-21. https://doi. org/10.1016/j.ejcts.2007.05.018. 
Diamond M, Feliciano H, Sanghavi D, Mahapatra S Acute Respiratory Distress Syndrome. In: Ncbi.nlm.nih.gov. 2020 https://www.ncbi. nlm.nih.gov/books/NBK436002/ Accessed 10 May 2020.

Diaz R, Heller D. Barotrauma and mechanical ventilation. StatPearls; 2020.

Díaz Lobato S, Mayoralas Alises S. La ventilación mecánica no invasiva moderna cumple 25 años. Arch Bronconeumol. 2013;49:475-9. https://doi.org/10.1016/j.arbres.2012.11.008.

DiBlasi R. Neonatal noninvasive ventilation techniques: do we really need to intubate? Respir Care. 2011;56:1273-97. https://doi.org/ 10.4187/respcare.01376.

Downs J, Klein E, Desautels D, et al. Intermittent mandatory ventilation: a new approach to weaning patients from mechanical ventilators. Chest. 1973;64:331-5. https://doi.org/10.1378/chest.64.3.331.

Duiverman M. Noninvasive ventilation in stable hypercapnic COPD: what is the evidence? ERJ Open Research. 2018;4:00012-2018. https://doi.org/10.1183/23120541.00012-2018.

Easton J, Wood M. Helmet-based ventilation is superior to face mask for patients with respiratory distress - UChicago medicine. In: Uchicagomedicine.org. 2020 https://www.uchicagomedicine.org/ forefront/patient-care-articles/helmet-based-ventilation-is-superiorto-face-mask-for-patients-with-respiratory-distress. Accessed 1 Jun 2020

Ellsbury D, Klein J, Segar J. Optimization of high-frequency oscillatory ventilation for the treatment of experimental pneumothorax. Crit Care Med. 2002;30:1131-5. https://doi.org/10.1097/00003246200205000-00029.

Engels P, Bagshaw S, Meier M, Brindley P. Tracheostomy: from insertion to decannulation. Can J Surg. 2009;52(5):427-33.

ESICM Academy. Mechanical Ventilation - COVID-19: Means to deliver positive pressure ventilation. In: Academy.esicm.org. 2020 https://academy.esicm.org/mod/emodulepage/view.php?id=7964. Accessed 2 Jun 2020.

Gibson P, Qin L, Puah S. COVID -19 acute respiratory distress syndrome (ARDS ): clinical features and differences from typical pre- COVID -19 ARDS. Med J Aust. 2020;213:54-56.e1. https://doi.org/10. 5694/mja2.50674.

Grieco D, Menga L, Raggi V, et al. Physiological comparison of highflow nasal cannula and helmet noninvasive ventilation in acute hypoxemic respiratory failure. Am J Respir Crit Care Med. 2020;201: 303-12. https://doi.org/10.1164/rccm.201904-0841oc.

Grum C, Morganroth M. Analytic reviews : initiating mechanical ventilation. J Intensive Care Med. 1988;3:6-20. https://doi.org/10.1177/ 088506668800300103

He Y, Li W, Wang Z, Chen H, Tian L, Liu D. Nosocomial infection among patients with COVID-19: a retrospective data analysis of 918 cases from a single center in Wuhan, China. Infect Control Hosp Epidemiol. 2020;41:982-3. https://doi.org/10.1017/ice.2020. 126.

Henzler D, Pelosi P, Bensberg R, Dembinski R, Quintel M, Pielen V, et al. Effects of partial ventilatory support modalities on respiratory function in severe hypoxemic lung injury*. Crit Care Med. 2006;34: 1738-45. https://doi.org/10.1097/01.ccm.0000218809.49883.54.

Hess D. Noninvasive positive-pressure ventilation and ventilatorassociated pneumonia. Respir Care. 2005;50(7):924-31.

Hess D, MacIntyre N, Galvin W, Mishoe S. Respiratory care: Jones \&Barlett Publishers; 2001.

Hong $\mathrm{C}, \mathrm{Xu} \mathrm{D}, \mathrm{Lu} \mathrm{Q}$, et al. Low tidal volume and high positive endexpiratory pressure mechanical ventilation results in increased inflammation and ventilator-associated lung injury in Normal lungs. Anesth Analg. 2010;110:1652-60. https://doi.org/10.1213/ane. 0b013e3181 cfc416.

Ioannidis G, Lazaridis G, Baka S, Mpoukovinas I, Karavasilis V, Lampaki S, et al. Barotrauma and pneumothorax. Journal of Thorac Disease. 2015;7(Suppl 1):S38-43. https://doi.org/10.3978/ j.issn.2072-1439.2015.01.31.
Jackson C. What is positive-pressure ventilation and how did its use develop for mechanical ventilation?. In: Medscape.com 2019 https://www.medscape.com/answers/304068-104771/what-ispositive-pressure-ventilation-and-how-did-its-use-develop-formechanical-ventilation. Accessed 30 May 2020.

Jackson C, Muthiah M. What is the background of the iron lung form of mechanical ventilation?. In: Medscape.com. 2019. https://www. medscape.com/answers/304068-104770/what-is-the-backgroundof-the-iron-lung-form-of-mechanical-ventilation. Accessed 5 Jun 2020.

Jounieaux V, Duran A, Levi-Valensi P. Synchronized intermittent mandatory ventilation with and without pressure support ventilation in weaning patients with COPD from mechanical ventilation. Chest. 1994;105:1204-10. https://doi.org/10.1378/chest.105.4.1204.

K M. Tracheostomy in ICU: an insight into the present concepts. Indian Journal of Anaesthesia. 2008;52:28.

Kacmarek R. The mechanical ventilator: past, present, and future. Respir Care. 2011;56:1170-80. https://doi.org/10.4187/respcare.01420.

Khandpur RS. Handbook of biomedical instrumentation. 3rd ed: McGraw Hill; 2003.

Kliff S, Satariano A, Silver-Greenberg J, Kulish N. There Aren't enough ventilators to cope with the coronavirus. In: Nytimes.com. 2020. https:/www.nytimes.com/2020/03/18/business/coronavirusventilator-shortage.html. Accessed 7 Jun 2020.

Kumar A, Pontoppidan H, Falke K, et al. Pulmonary barotrauma during mechanical ventilation. Crit Care Med. 1973;1:181-6. https://doi. org/10.1097/00003246-197307000-00001.

Lazoff S, Kim B. Synchronized intermittent mandatory ventilation. StatPearls; 2019.

Lemyre B, Laughon M, Bose C, Davis P. Early nasal intermittent positive pressure ventilation (NIPPV) versus early nasal continuous positive airway pressure (NCPAP) for preterm infants. Cochrane Database Syst Rev. 2016. https://doi.org/10.1002/14651858.cd005384.pub2.

Lettieri C, Williams S, Collen J, Wickwire E. Treatment of obstructive sleep apnea. Sleep Med Clin. 2020;15:227-40. https://doi.org/10. 1016/j.jsmc.2020.02.009.

Li L, Li R, Wu Z, Yang X, Zhao M, Liu J, et al. Therapeutic strategies for critically ill patients with COVID-19. Ann Intensive Care. 2020a;10:45. https://doi.org/10.1186/s13613-020-00661-z.

Li J, Fink J, Ehrmann S. High-flow nasal cannula for COVID-19 patients: low risk of bio-aerosol dispersion. Eur Respir J. 2020b;55:2000892. https://doi.org/10.1183/13993003.00892-2020.

Lucangelo U, Pelosi P, Zin W, Aliverti A Respiratory system and artificial ventilation. Springer; 2008.

McGrath B, Brenner M, Warrillow S, et al. Tracheostomy in the COVID19 era: global and multidisciplinary guidance. Lancet Respir Med. 2020;8:717-25. https://doi.org/10.1016/s2213-2600(20)30230-7.

McMullen S, Meade M, Rose L, et al. Partial Ventilatory support modalities in acute lung injury and acute respiratory distress syndrome - a systematic review. PLoS One. 2012;7:e40190. https://doi.org/10. 1371/journal.pone.0040190.

Moerer O, Harnisch L. Non-invasive mechanical ventilation in hypoxemic respiratory failure: just a matter of the interface? Journal of thoracic disease. 2016;8:2348-52. https://doi.org/10.21037/jtd. 2016.08.43.

Nicolini A, Banfi P, Grecchi B, Lax A, Walterspacher S, Barlascini C, et al. Non-invasive ventilation in the treatment of sleep-related breathing disorders: a review and update. Rev Port Pneumol. 2014;20:324-35. https://doi.org/10.1016/j.rppneu.2014.03.009.

NIH Oxygenation and Ventilation | COVID-19 Treatment Guidelines. In: COVID-19 Treatment Guidelines. 2020. https://www. covid19treatmentguidelines.nih.gov/critical-care/oxygenation-andventilation/. Accessed 12 Jun 2020.

Nishimura M. High-flow nasal cannula oxygen therapy in adults. J Intensive Care. 2015;3:15. https://doi.org/10.1186/s40560-0150084-5. 
Oeckler R, Hubmayr R. Ventilator-associated lung injury: a search for better therapeutic targets. Eur Respir J. 2007;30:1216-26. https:// doi.org/10.1183/09031936.00104907.

Patel B, Wolfe K, Pohlman A, et al. Effect of noninvasive ventilation delivered by helmet vs face mask on the rate of endotracheal intubation in patients with acute respiratory distress syndrome. JAMA. 2016;315:2435-41. https://doi.org/10.1001/jama.2016.6338.

Patel B, Wolfe K, MacKenzie E, et al. One-year outcomes in patients with acute respiratory distress syndrome enrolled in a randomized clinical trial of helmet versus facemask noninvasive ventilation. Crit Care Med. 2018;46:1078-84. https://doi.org/10.1097/ccm. 0000000000003124

Pesenti A, Patroniti N. Low tidal volume, high respiratory rate and autoPEEP: the importance of the basics. Crit Care. 2003;7(2):105-6. https://doi.org/10.1186/cc1883.

Pi X, Cui Y, Wang C, Guo L, Sun B, Shi J, et al. Low tidal volume with PEEP and recruitment expedite the recovery of pulmonary function. Int J Clin Exp Pathol. 2015;8(11):14305-14.

Pinto V, Sharma S. Continuous positive airway pressure. StatPearls; 2020.

Poor H. Basics of mechanical ventilation: Springer; 2018.

Pupella R. Mechanical ventilation in patient with respiratory failure. Springer Singapore, Imprint: Springer, Singapore; 2018.

Ranu H, Wilde M, Madden B. Pulmonary function tests. Ulster Med J. 2011;80(2):84-90.

Rashid A, Islam S. Percutaneous tracheostomy: a comprehensive review. Journal of thoracic disease. 2017;9:S1128-38. https://doi.org/10. 21037/jtd.2017.09.33.

Raymondos K, Molitoris U, Capewell M, Sander B, Dieck T, Ahrens J, et al. Negative- versus positive-pressure ventilation in intubated patients with acute respiratory distress syndrome. Crit Care. 2012;16: R37. https://doi.org/10.1186/cc11216.

Sahetya S, Goligher E, Brower R. Fifty years of research in ARDS.Setting positive end-expiratory pressure in acute respiratory distress syndrome. Am J Respir Crit Care Med. 2017;195:1429-38. https://doi.org/10.1164/rccm.201610-2035ci.

Scala R, Pisani L. Noninvasive ventilation in acute respiratory failure: which recipe for success? Eur Respir Rev. 2018;27:180029. https:// doi.org/10.1183/16000617.0029-2018.

Shneerson J. Assisted ventilation. 5. Non-invasive and domiciliary ventilation: negative pressure techniques. Thorax. 1991;46:131-5. https://doi.org/10.1136/thx.46.2.131.

Siegel M, Siemieniuk R. Acute respiratory distress syndrome: supportive care and oxygenation in adults. In: Uptodate.com $2020 \mathrm{https}: / \mathrm{www}$. uptodate.com/contents/acute-respiratory-distress-syndromesupportive-care-and-oxygenation-in-adults\#H20. Accessed 7 May 2020.

Silversides J, Ferguson N. Clinical review: acute respiratory distress syndrome - clinical ventilator management and adjunct therapy. Crit Care. 2013;17:225. https://doi.org/10.1186/cc11867.

Singh P, Ramasethu R, Sharma A. Prone ventilation and critical care management of severe ARDS and multiorgan failure in a young patient. Medical Journal Armed Forces India. 2014;70:85-8. https://doi.org/10.1016/j.mjafi.2012.08.016.

Slutsky A. History of mechanical ventilation. From Vesalius to Ventilator-induced Lung Injury. Am J Respir Crit Care Med. 2015;191:1106-15. https://doi.org/10.1164/rccm.201503-0421pp.
Soni N, Williams P. Positive pressure ventilation: what is the real cost? $\mathrm{Br}$ J Anaesth. 2008;101:446-57. https://doi.org/10.1093/bja/aen240.

Spicuzza L, Caruso D, Di Maria G. Obstructive sleep apnoea syndrome and its management. Therapeutic Advances in Chronic Disease. 2015;6:273-85. https://doi.org/10.1177/2040622315590318.

Spieth P, Carvalho A, Pelosi P, et al. Variable tidal volumes improve lung protective ventilation strategies in experimental lung injury. Am J Respir Crit Care Med. 2009;179:684-93. https://doi.org/10.1164/ rccm.200806-975oc.

Tobin M. Principles and practices of mechanical ventilation, 3rd ed. McGraw Hill Professional; 1994.

Tsolaki V, Siempos I, Magira E, Kokkoris S, Zakynthinos GE, Zakynthinos S. PEEP levels in COVID-19 pneumonia. Crit Care. 2020;24:303. https://doi.org/10.1186/s13054-020-03049-4.

USFDA. Enforcement policy for ventilators and accessories and other respiratory devices during the coronavirus disease 2019 (COVID19). In: U.S. Food and Drug Administration. $2020 \mathrm{https} / / \mathrm{www} . f d a$. gov/emergency-preparedness-and-response/mcm-issues/ coronavirus-disease-2019-covid-19. Accessed 3 Jun 2020.

van Doremalen N, Bushmaker T, Morris D, et al. Aerosol and surface stability of SARS-CoV-2 as compared with SARS-CoV-1. N Engl J Med. 2020;382:1564-7. https://doi.org/10.1056/nejmc2004973.

Walter J, Corbridge T, Singer B. Invasive mechanical ventilation. South Med J. 2018;111:746-53. https://doi.org/10.14423/smj. 0000000000000905 .

Wang K, Zhao W, Li J, Shu W, Duan J. The experience of high-flow nasal cannula in hospitalized patients with 2019 novel coronavirusinfected pneumonia in two hospitals of Chongqing. China Annals of Intensive Care doi. 2020;10:37. https://doi.org/10.1186/s13613020-00653-z

West J. The physiological challenges of the 1952 Copenhagen poliomyelitis epidemic and a renaissance in clinical respiratory physiology. J Appl Physiol. 2005;99:424-32. https://doi.org/10.1152/ japplphysiol.00184.2005.

WHO. Clinical management of severe acute respiratory infection (SARI) when COVID-19 disease is suspected: interim guidance, 13 march 2020. In: apps.who.int. 2020a. https://apps.who.int/iris/handle/ 10665/331446. Accessed 28 May 2020.

WHO. Modes of transmission of virus causing COVID-19: implications for IPC precaution recommendations. In: Who.int. 2020b. https:// www.who.int/news-room/commentaries/detail/modes-oftransmission-of-virus-causing-covid-19-implications-for-ipcprecaution-recommendations. Accessed 28 May 2020.

Yagui A, Vale L, Haddad L, et al. Bubble CPAP versus CPAP with variable flow in newborns with respiratory distress: a randomized controlled trial. J Pediatr. 2011;87:499-504. https://doi.org/10. 2223/jped.2145.

Yarstev A. Advantages and limitations of non-invasive ventilation | deranged physiology. In: Derangedphysiology.com. 2015. https:// derangedphysiology.com/main/cicm-primary-exam/requiredreading/respiratory-system/Chapter\%20526/advantages-andlimitations-non. Accessed 2 Jun 2020.

Publisher's note Springer Nature remains neutral with regard to jurisdictional claims in published maps and institutional affiliations. 